\title{
Oxidative stress parameters in paediatric non-alcoholic fatty liver disease
}

\author{
VALERIO NOBILI $^{1}$, MAURIZIO PAROLA $^{5}$, ANNA ALISI $^{1}$, FABIO MARRA $^{3}$, FIORELLA PIEMONTE $^{2}$, \\ CRISTINA MOMBELLO ${ }^{4}$, SALVATORE SUTTI $^{4}$, DAVIDE POVERO ${ }^{5}$, VIRGINIA MAINA ${ }^{4}$, \\ ERICA NOVO 5 and EMANUELE ALBANO ${ }^{4}$
}

\begin{abstract}
Departments of ${ }^{1}$ Hepatogastroenterology; ${ }^{2}$ Molecular Medicine Bambino Gesù Children's Hospital, Rome; ${ }^{3}$ Department of Internal Medicine, University of Florence, Florence; ${ }^{4}$ Department of Medical Sciences, University 'A. Avogadro' of East Piedmont, Novara; ${ }^{5}$ Department of Experimental Medicine and Oncology, University of Turin, Turin, Italy
\end{abstract}

Received April 8, 2010; Accepted May 28, 2010

DOI: 10.3892/ijmm_00000487

\begin{abstract}
We have investigated the presence and the possible clinical implications of oxidative stress in children with nonalcoholic fatty liver disease (NAFLD). The present study was an observational study of oxidative stress parameters in the progression of paediatric NAFLD. We observed the role of oxidative stress in children diagnosed with NAFLD by evaluating: serum protein carbonyls, hepatic expression of 8-hydroxy-2-deoxyguanosine (8-OHG), and circulating antibody against malondialdehyde adducted human serum albumin (MDA-HSA). Forty consecutive children with biopsy-proven NAFLD (27 male; 13 female) referred to Bambino Gesù Children's Hospital, Rome, Italy, from January 2007 to April 2008 were included in the study. Serum variations of protein carbonyls, 8-OHG, and circulating antibody against MDAHSA were evaluated. Elevated protein carbonyls were evident in 33 subjects (83\%) irrespective of obesity and insulin resistance. Moreover, liver biopsies of NAFLD patients positive for circulating protein carbonyls also showed a significant increase in the nuclear staining for 8-OHG $(\mathrm{p}=0.006$; 95\% CI 3.1-17.7). Anti-MDA-HSA IgG above control threshold was detected in $25(63 \%)$ children. Although protein carbonyl levels were unrelated with disease severity, patients with elevated anti-MDA-HSA IgG had scores for lobular inflammation significantly higher $(p=0.019)$ than subjects with antibodies within the control range, while steatosis, hepatocyte ballooning and fibrosis were similar. High anti-MDA-HSA reactivity was also associated with a 13 -fold increased risk $(\mathrm{OR}=12.9 ; 95 \% \mathrm{CI}$
\end{abstract}

Correspondence to: Dr Valerio Nobili, Dipartimento di Epatogastroenterologia e Nutrizione, Ospedale Pediatrico Bambino Gesù, Piazza S. Onofrio 4, I-00165 Roma, Italy

E-mail: nobili66@yahoo.it

Key words: oxidative stress, non-alcoholic fatty liver disease, nonalcoholic steatohepatitis, non-alcoholic fatty liver disease activity score
$1.5-113.8 ; \mathrm{p}=0.013)$ of a NAFLD activity score $(\mathrm{NAS}) \geq 5$. These results demonstrate that oxidative stress has an high prevalence in children with NAFLD and is associated with an increased severity of steatohepatitis.

\section{Introduction}

Paediatric non-alcoholic fatty liver disease (NAFLD) has become the most frequent chronic liver disease in children and adolescents of industrialized countries due to the growing prevalence of childhood obesity and overweight (1-3). Presently, NAFLD prevalence among children and adolescents ranges between $2.6-9.8 \%$ and is especially high among obese subjects. Such a high prevalence of NAFLD and the likelihood of evolution to cirrhosis warrant increased attention toward this disease $(4,5)$. NAFLD progression toward hepatic fibrosis and cirrhosis depends upon the combination of necroinflammation and increased fibrogenesis that characterize non-alcoholic steatohepatitis (NASH). In addition, obesity and older age are independently associated with more advanced fibrosis (6). Thus, a key issue in unravelling the pathogenesis of NASH is the characterization of the mechanisms promoting necro-inflammation and healing responses in fatty livers.

Increasing evidence from experimental models of NAFLD/NASH suggests that oxidative stress plays a key role in mechanisms leading to the death of fat-laden hepatocytes and contributes to activating hepatic stellate cells to matrixproducing myofibroblasts. Moreover, redox changes are responsible for increasing Kupffer cell response to proinflammatory stimuli $(7,8)$. Accordingly, antioxidant supplementation reduces liver injury in experimental rodent models of NASH $(9,10)$. The relevance of these observations in humans is supported by several studies showing increased liver and serum content of oxidative stress markers, such as lipid peroxidation end-products, 8-hydroxy-2-deoxyguanosine (8-OHG), and nitrotyrosine in adult patients with NAFLD/ NASH (11-13). Moreover, microarray analysis of liver biopsies from patients with NASH demonstrated lower mRNA expression of different antioxidant enzymes (14). However, information regarding the involvement of oxidative stress in paediatric NAFLD has been, to date, quite scattered (2). This is 
an important limitation considering that antioxidant supplementation is under evaluation for the therapy of paediatric NAFLD $(15,16)$.

In the present study we investigated the presence and the possible clinical implications of oxidative stress in children with NAFLD/NASH.

\section{Patients and methods}

Patients. A total of 40 consecutive patients diagnosed with NAFLD (27 male; 13 female) referred to Bambino Gesù Children's Hospital, Rome, Italy, from January 2007 to April 2008 were included in the study. Inclusion criteria were, persistently elevated serum aminotransferase levels, diffusely echogenic liver or imaging studies suggestive of fatty liver and biopsy consistent with the diagnosis of NAFLD (15-17). Exclusion criteria were hepatic virus infections (Hepatitis A, B, C, D, E and G, cytomegalovirus and Epstein-Barr virus), alcohol consumption, history of parenteral nutrition, and use of drugs known to induce steatosis (e.g. valproate, amiodarone or prednisone) or to affect body weight and carbohydrate metabolism. Autoimmune and metabolic liver disease, Wilson's disease, and $\alpha$-1-antitrypsin-associated liver disease were ruled out using standard clinical, laboratory and histological criteria. Informed consent was obtained from each patient or responsible guardian. The study was approved by the Ethics Committee of the Bambino Gesù Children's Hospital and Research Institute, Rome, Italy.

The body mass index (BMI) and BMI Z-score were calculated according to established criteria $(18,19)$. Metabolic syndrome was defined by the presence of three or more of the following five criteria: abdominal obesity (defined by waist circumference $\geq 90$ th percentile for age), hypertriglyceridemia (triglycerides $>95$ th percentile for age, gender and race), low HDL cholesterol (HDL cholesterol $<5$ th percentile for age and gender), elevated blood pressure (defined as systolic or diastolic blood pressure $>95$ th percentile for age and gender), and impaired fasting glucose or known type 2 diabetes mellitus.

A 2-h oral glucose tolerance test (OGTT) was performed with the standard $1.75 \mathrm{~g}$ of glucose per $\mathrm{kg}$ of body weight, or a maximum of $75 \mathrm{~g}$. Glucose tolerance status was determined according to the classification of the American Diabetes Association in which fasting plasma glucose levels up to $99 \mathrm{mg} / \mathrm{dl}$ are considered normal; impaired fasting glucose (IFG) is defined by a fasting plasma glucose of $100-125 \mathrm{mg} / \mathrm{dl}$; impaired glucose tolerance (IGT) is defined by a 2 -h plasma glucose of $140-199 \mathrm{mg} / \mathrm{dl}$; diabetes mellitus is defined by a fasting plasma glucose $\geq 126 \mathrm{mg} / \mathrm{dl}$, or a 2-h plasma glucose $\geq 200 \mathrm{mg} / \mathrm{dl}(20,21)$. Plasma glucose was measured in triplicate by the glucose oxidase technique on a Beckman glucose analyzer (Beckman, Fullerton, CA) and plasma insulin was measured by a specific radioimmunoassay (Myria Technogenetics, Milan, Italy). The degrees of insulin resistance and sensitivity were determined, respectively, by the homeostatic model assessment insulin resistance (HOMA-IR) using the formula: $\mathrm{IR}=($ insulin $\mathrm{x}$ glucose $) / 22.5$; and by the insulin sensitivity index (ISI) derived from OGTT using the formula: ISI $=[10,000 /$ square root of (fasting glucose $\mathrm{x}$ fasting insulin) $\mathrm{x}$ (mean glucose $\mathrm{x}$ mean insulin during OGTT)] $(22,23)$.
Liver histology. Liver biopsy was performed in all NAFLD children, after an overnight fast, using an automatic core biopsy 18 Gauge needle (Biopince, Amedic, Sweden) under general anaesthesia and ultrasound guidance. A Sonoline Omnia Ultrasound machine (Siemens, Germany) with a 5-MHz probe (5.0 C 50, Siemens) was employed. Two biopsy passes within different liver segments were performed for each subject. The length of the liver specimen (in $\mathrm{mm}$ ) was recorded. Only samples with a length $\geq 15 \mathrm{~mm}$ and including at least 10-11 complete portal tracts were considered adequate for the purpose of the study (24). Biopsies were evaluated by a single liver pathologist. Sections of liver tissue, $5 \mu \mathrm{m}$ thick, were stained with hematoxylin-eosin, Van Gieson, PAS-D, and Prussian blue staining. Immunohistochemical staining with antibodies against $\alpha$-1-anti-trypsin was used to exclude $\alpha-1$ anti-trypsin deficiency-associated liver disease. Liver biopsy features were graded according to the NAFLD activity scoring (NAS) system proposed by Kleiner et al (25). Briefly, grade of steatosis was scored as $0,<5 \% ; 1,5-33 \%$; $2,>33-66 \%: 3,>66 \%$; grade of lobular inflammation was scored as 0 , no foci; 1, <2 foci/x200 field; 2, 2-4 foci/x200 field; 3, >4 foci/x200 field; and grade of ballooning was scored as 0 , none; 1 , few ballooning cells; 2 , many cells/ prominent ballooning. The grade of steatosis (0-3), lobular inflammation (0-3), and ballooning (0-2) were then combined to determine the NAFLD activity score (0-8). Fibrosis was scored as 0 , none; 1 , periportal or perisinusoidal fibrosis; 2 , perisinusoidal and portal/periportal fibrosis; 3, bridging fibrosis; and 4, cirrhosis. Fibrosis score for stage 1 was extended to include a distinction between delicate perisinusoidal fibrosis (stage $1 \mathrm{~A}$ ), dense perisinusoidal fibrosis (stage 1B), and portal-only fibrosis without perisinusoidal fibrosis (stage 1C) (25).

Assessment of oxidative stress in NAFLD children. Serum levels of protein carbonyls were measured by reactions with 2,4 dinitrophenylhydrazine using the Protein Carbonyls Assay Kit (Cayman Chemical Company, Ann Arbor, MI, USA) according to the manufacturer's instructions. Serum samples from 18 normal-weight children matched for gender and age and without echographic evidence of fatty liver undergoing routine surgery were used as controls. Threshold values were calculated as 95th percentile in the controls. Oxidative injury was confirmed by the immunohistochemical detection of 8-OHG in paraffin-embedded liver biopsies using a monoclonal anti-8-OHdG antibody (Japanese Aging Control Institute, Shizuoka, Japan) (final dilution $5 \mu \mathrm{g} / \mathrm{ml}$ ) as previously described (26). The percentage of stained hepatocyte nuclei was evaluated in a blinded manner in ten different microscopical fields.

Measurement of antibody titres against malondialdehydederived adducts. MDA adducted with human serum albumin (MDA-HSA) was prepared by reacting for $2 \mathrm{~h}$ at $37^{\circ} \mathrm{C} 2 \mathrm{mg} / \mathrm{ml}$ HSA with $100 \mathrm{mmol} / \mathrm{l} \mathrm{MDA}$ as previously reported (27). For the evaluation of immune response induced by oxidative stress polystyrene microwell plates for enzyme-linked immunosorbent assay (ELISA) (Nunc-Immuno Maxi-Sorb, Nunc, $\mathrm{S} / \mathrm{A}$, Roskilde, Denmark) were coated for $4 \mathrm{~h}$ at $37^{\circ} \mathrm{C}$ with $0.05 \mathrm{mg} / \mathrm{ml}$ of either modified or native HSA solubilised in 
Table I. Clinical and biochemical characterization of NAFLD children investigated.

\begin{tabular}{lrr}
\hline Parameters & \multicolumn{2}{c}{ Patients } \\
\hline Patients (male/female) & 40.0 & $(27 / 13)$ \\
Age (years) & 11.57 & $(10.04-12.89)$ \\
BMI & 24.6 & $(22.7-28.9)$ \\
HOMA-IR (n.v. <3) & 2.18 & $(1.61-3.06)$ \\
ISI (n.v. >6) & 4.21 & $(3.13-5.70)$ \\
AST (U/l - n.v. 5-40) & 60.0 & $(45.24-78.0)$ \\
ALT (U/l - n.v. 5-40) & 44.0 & $(32.0-54.50)$ \\
$\gamma$-GT (U/l - n.v. 5-45) & 18.5 & $(15.75-34.25)$ \\
Fasting glucose (mg/dl - n.v. <100) & 83.0 & $(76.0-91.25)$ \\
Cholesterol (mg/dl - n.v. <200) & 149.5 & $(130-176)$ \\
Triglycerides (mg/dl - n.v. <160) & 80.0 & $(59.0-99.25)$ \\
Steatosis score & 2.0 & $(1-3)$ \\
Inflammation score & 1.0 & $(0-3)$ \\
Ballooning score & 0.0 & $(0-2)$ \\
Fibrosis score & 1.0 & $(0-3)$ \\
NAS score & 4.0 & $(1-6)$ \\
\hline
\end{tabular}

Values are expressed as median and inter-quartile range (IQR). For histological scores the range of variability is included. BMI, body mass index; AST, alanine aminotransferase; ALT, aspartate aminotransferase; $\gamma$-GT, $\gamma$-glutamyl transpeptidase; HOMA-IR, homeostatic model assessment-insulin resistance; ISI, insulin sensitivity index; n.v., normal values; NAS, NAFLD activity score.

0.1 M bicarbonate buffer, $\mathrm{pH}$ 9.6. After incubation, solutions were removed and replaced by $0.3 \mathrm{ml}$ of coating buffer containing $3 \%$ bovine serum albumin (BSA) in phosphate buffered saline (PBS), $\mathrm{pH}$ 7.4. Plates were further incubated for $1 \mathrm{~h}$ at $37^{\circ} \mathrm{C}$ to block non-specific binding sites. Coated wells were washed three times with PBS containing $0.25 \%$ Triton X-100. Human sera $(0.20 \mathrm{ml}$, dilution $1: 50$ in the coating buffer) were added in duplicate and incubated for $1 \mathrm{~h}$ at $37^{\circ} \mathrm{C}$. After washing three times with PBS-0.25\% Triton $\mathrm{X}-100$, antibody binding was revealed using peroxidaselinked goat anti-human IgG (dilution 1:6,000) (Dako S.P.A., Milano, Italy) as previously described (27). The results were expressed by subtracting background reactivity from unmodified HSA. Threshold values were calculated as 95th percentile in the control population.

Data analysis and statistical calculations. Statistical analyses were performed by SPSS statistical software (SPSS Inc. Chicago IL, USA) using one-way ANOVA or KruskalWallis test for non-parametric values. Confidence intervals were calculated using the CIA software (by T. Bryant, University of Southampton, UK). Relative risk and Fisher's Exact Tests were used for the comparison of frequency data. Significance was taken at the 5\% level. The independent effect of significant variables was assessed using stepwise logistic regression analysis. Normality distribution was preliminary assessed by the Kolmogorov-Smirnov and the Shapiro-Wilk tests and corrections were performed by logarithmic transformation.

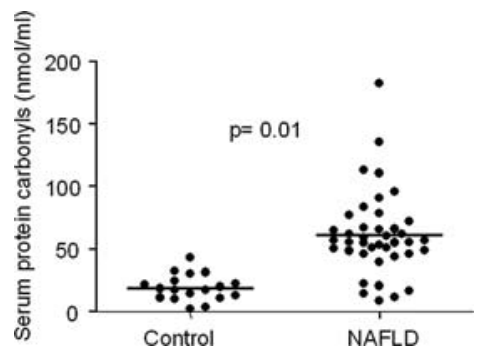

Figure 1. Individual levels of serum protein carbonyls in 40 children with NAFLD and 18 age and gender matched controls. Horizontal bars represent medians.

\section{Results}

For this study we enrolled 40 paediatric biopsy-proven NAFLD patients (27 male; 13 female), mean age 11.5 years (range, 5.8-19.1 years). The major clinical and biochemical parameters are reported in Table I. Obesity was present in 25 subjects $(62 \%)$, while insulin resistance, as estimated by HOMA-IR and ISI indexes, was detected in 30 subjects (75\%). Hypercholesterolemia and hypertriglyceridemia were observed in 1 patient (3\%), respectively. The presence of NAFLD was confirmed histopathologically in all subjects. Extensive steatosis ( $>66 \%)$ was evident in 16 subjects $(40 \%)$. Twenty-six patients $(65 \%)$ showed mild-moderate perisinusoidal or periportal fibrosis, $1(3 \%)$ had perisinusoidal and portal/periportal fibrosis and $3(8 \%)$ bridging fibrosis.

Serum protein carbonyls were significantly increased in NAFLD patients $(61.0 \pm 33.4$ vs $20.7 \pm 13.8 \mathrm{nmol} / \mathrm{ml} ; \mathrm{p}<0.0001$ 95\% CI 27.7-52.8) as compared to 18 gender- and agematched healthy controls (Fig. 1). Furthermore, protein carbonyl values above the control threshold were evident in 33 subjects $(83 \%)$. Protein carbonyl levels were unrelated with age, gender, obesity, insulin resistance, circulating triglyceride and glucose or the biochemical or histopathological severity of the disease (data not shown). Immunohistochemistry 8-OHG, a marker of oxidative DNA damage confirmed the liver specificity of oxidative damage, as 8-OHG nuclear staining was significantly higher $(\mathrm{p}=0.006 ; 95 \%$ CI $3.1-17.7)$ in liver biopsies from NAFLD patients positive for circulating protein carbonyls than in those who were negative (Fig. 2).

Recent data from a rodent model of NAFLD indicate that hepatic oxidative stress leads to the development of an antibody response against protein modified by the reaction with end-products of lipid peroxidation, namely malondialdehyde (MDA) (10). Similar antibodies have also been detected in adult NAFLD patients and are an independent predictor for advanced fibrosis (28). These observations prompted us to investigate whether antibodies against MDA-derived antigens were associated with paediatric NAFLD. As shown in Fig. 2, 25 out of 40 NAFLD patients $(63 \%)$ had circulating IgG against MDA adducts with human serum albumin (MDAHSA) above the control threshold. The presence of antiMDA-HSA IgG was not influenced by age, gender, obesity, insulin resistance, aminotransferase or GGT release (data not shown). However, at the histology, patients with elevated antiMDA-HSA antibodies showed that scores of lobular inflammation were significantly higher $(p=0.019)$ than subjects with antibodies within control range (Fig. 3). Lobular 

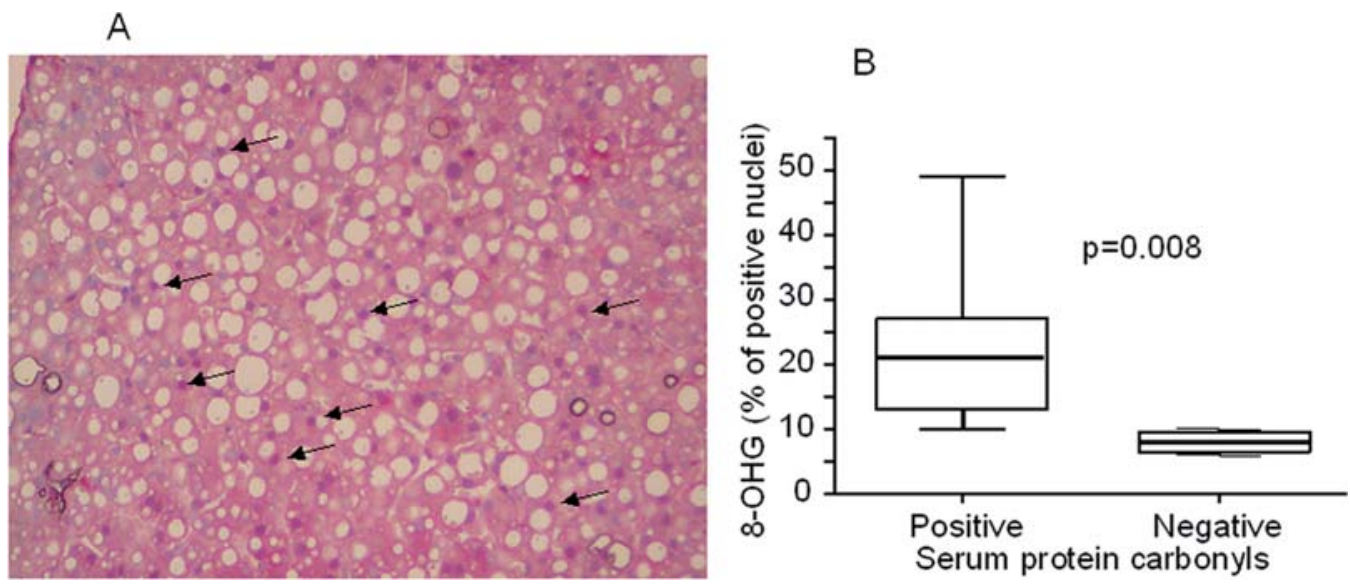

Figure 2. Immunohistochemical detection of 8-hydroxydeoxyguanosine (8-OHG) in liver biopsies form NAFLD children. (A) Predominant localization of 8-OHG in the nuclei of parenchymal cells is indicated by arrows. (B) Difference in the percentage of 8-OHG-positive nuclei in liver biopsies from NAFLD children with or without increased circulating protein carbonyls. Boxes include values within 25 th and 75 th percentile, while horizontal bars represent medians. Eighty percent of the values are comprised between the extremities of the vertical bars (10th-90th percentile).

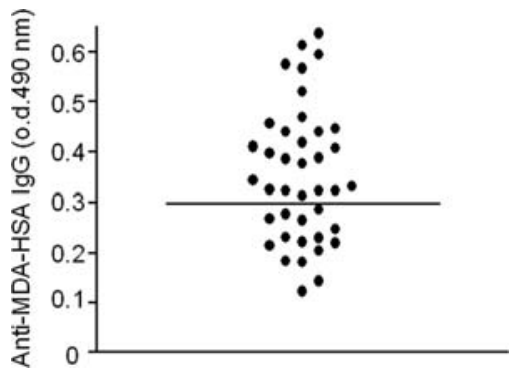

Figure 3. Distribution of the individual IgG reactivity against malondialdehydemodified human serum albumin (MDA-HSA) among 40 children with NAFLD. Results are expressed as optical density (o.d.) at $490 \mathrm{~nm}$, as reported in Patients and methods. Horizontal bars represent threshold values calculated at the 95 th percentile in a control population.
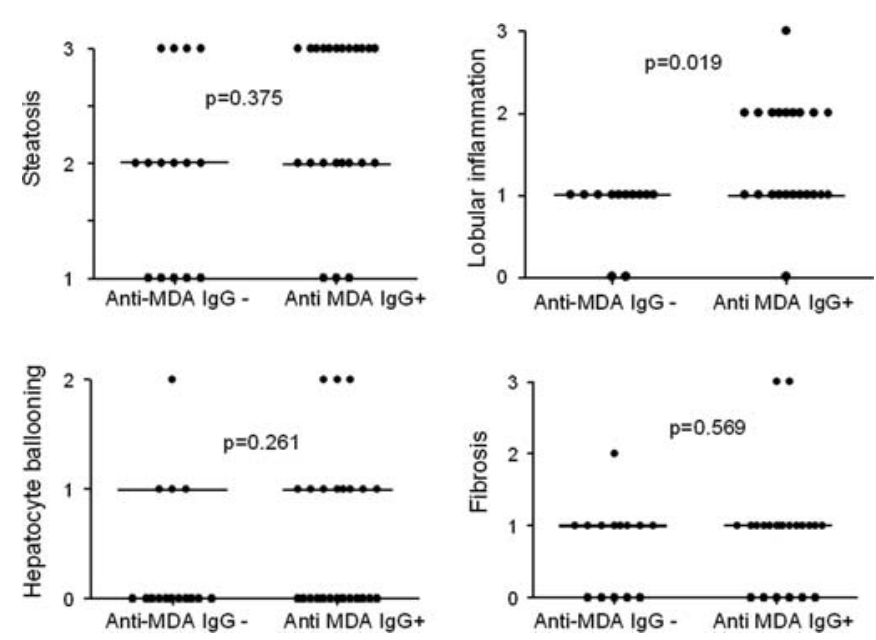

Figure 4. Distribution of the individual histological score for steatosis, lobular inflammation, hepatocyte ballooning and fibrosis among 40 children with NAFLD sub-grouped according to the presence (Anti-MDA $\operatorname{IgG}^{+}$) or absence (Anti-MDA $\operatorname{IgG}^{-}$) of elevated $\mathrm{IgG}$ reactivity against malondialdehyde-modified human serum albumin. Horizontal bars represent medians.

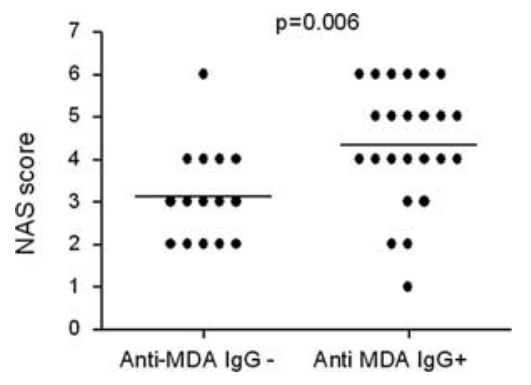

Figure 5. Distribution of the individual values of NAFLD activity score (NAS) among 40 children with NAFLD sub-grouped according to the presence (Anti-MDA $\mathrm{IgG}^{+}$) or absence (Anti-MDA $\mathrm{IgG}^{-}$) of elevated $\mathrm{IgG}$ reactivity against malondialdehyde-modified human serum albumin. Horizontal bars represent median.

inflammation score $>1$ was also prevalent among NAFLD children with elevated anti-MDA-HSA $\operatorname{IgG}(0 \%$ vs $40 \%$; $\mathrm{p}=0.006 ; 95 \%$ CI 1.2-2.3). No significant differences were observed in the extension of steatosis, frequency of hepatocyte ballooning or severity of fibrosis (Fig. 4). NAFLD activity score (NAS) was higher (median 4; range 1-6) in NAFLD patients with anti-MDA-HSA antibodies as compared to the remaining patients (median 3; range 2-6; p=0.006) (Fig. 5). Moreover, a NAS score $\geq 5$, considered indicative of overt $\mathrm{NASH}, 25$ was 13 -fold more frequent $(\mathrm{OR}=12.9$; 95\%CI 1.5113.8; $\mathrm{p}=0.013$ ) among subjects with elevated anti-MDAHSA IgG.

\section{Discussion}

Several studies have implicated oxidative stress in adult NAFLD/NASH (11-13), however, very little is known about the involvement of oxidative damage in paediatric NAFLD (2). Our observations demonstrate that a high proportion $(83 \%)$ of children with NAFLD show signs of oxidative injury, as evaluated by elevated circulating levels of protein carbonyls. Protein-bound carbonyls arise from free radical-mediated 
protein oxidation and are a widely used generic marker of oxidative stress (29). Although protein carbonyls can also originate from protein glycoxidation, elevated serum protein carbonyls are unrelated to glycemia or insulin resistance, indicating that they are expression of oxidative injury (29). Children with elevated serum protein carbonyls also show an increased hepatocyte nuclear staining for $8-\mathrm{OHG}$, a marker of oxidative DNA damage. Interestingly, 8-OHG presence in parenchymal cells along with cytosolic accumulation of the lipid peroxidation product 4-hydroxynonenal have been documented in adult NASH (11). Thus, circulating protein carbonyls in NAFLD children likely reflect hepatic oxidative injury consequent to fat accumulation. Indeed, mitochondrial alterations caused by hepatocyte free fatty acid overload along with the induction of cytochrome P450 2E1 (CYP2E1) are regarded as the main causes for oxidative injury in NAFLD (8). In agreement with such an interpretation, previous reports have shown that paediatric NAFLD is characterized by a decrease in the plasma antioxidant content and by an abnormal glutathione metabolism (30-32).

In a significant fraction of NAFLD children $(63 \%)$ hepatic oxidative injury is associated with the development of antibodies towards protein adducted by malonildialdehyde (MDA). Similar antibodies have also been observed in $\sim 40 \%$ of adult NAFLD patients, as well as in subjects with advanced alcoholic liver disease (ALD) and chronic hepatitis C, two other liver diseases characterized by the involvement of oxidative stress (26,27,33-36). Moreover, $35 \%$ of ALD patients also have circulating $\mathrm{CD}^{+}{ }^{+} \mathrm{T}-\mathrm{lym}$ hhocytes recognizing MDA adducts, indicating that oxidative mechanisms can promote both humoral and cellular immune responses (37).

At present the mechanisms responsible for oxidative stress-driven immunity in NAFLD are still poorly understood. The phagocytosis of oxidatively-modified hepatic proteins by dendritic cells in intraportal lymphoid follicles might lead to the priming of naïve B and T cells (38). This process is likely facilitated by the capacity of the scavenger receptors and of some pattern recognition receptors to recognize oxidativelymodified proteins and lipids (39). An additional factor in the development of immune response in NAFLD may involve the impairment of immuno-regulatory mechanisms, such as steatosis and oxidative stress lower hepatic regulatory $\mathrm{T}$ cells (Tregs) in mice fed a high fat diet (40). Indeed, Treg lowering is now regarded as an important event in promoting lymphocyte activation against oxidized LDL during the evolution of atherosclerosis (41).

Previous studies have shown that the intra-hepatic detection of lipid peroxidation products is prevalent in patients with NASH and correlates with necro-inflammation (11-13). Several lines of evidence in ALD indicate the involvement of immunological mechanisms in fuelling hepatic inflammation during the progression of the disease (27). In particular, we have observed that heavy drinkers with high circulating levels of oxidative stress-induced antibodies have a 5-fold higher prevalence of elevated plasma TNF- $\alpha$ levels and 11-fold greater risk of advanced ALD than heavy drinkers with these antibodies within the control range (26). In the present study, NAFLD children with immune responses against MDAderived antigens show more severe lobular inflammation and have a 13-fold higher prevalence of overt NASH (NAS score $\geq 5$ ). Conversely, no association is evident between elevated serum protein carbonyls and liver histology. Altogether these results suggest that the activation of adaptive immunity against defined oxidative stress-derived antigens contributes to hepatic inflammation in pediatric NAFLD. In contrast to adult NAFLD, we did not observe any significant association between the presence of anti-MDA-HSA antibodies and the severity of fibrosis. This might be due to the low prevalence of extensive fibrosis in the population sample investigated (27).

In conclusion, these results demonstrate that oxidative stress is present in a high proportion of NAFLD children and leads to the development of lipid peroxidation-related antibodies that are associated with elevated NAS scores. If confirmed by prospective studies, the evaluation of $\operatorname{IgG}$ reactivity towards MDA adducts may become a useful marker for the identification of NAFLD children at risk of disease progression who might benefit from targeted antioxidant therapy.

\section{Acknowledgements}

This study was supported by grants from Ospedale Bambino Gesù in Rome and the Regional Government of Piedmont (Progetto Ricerca Sanitaria Finalizzata 2008-bis).

\section{References}

1. Reilly JJ: Obesity in childhood and adolescence: evidence-based clinical and public health perspectives. Postgrad Med J 82: 429-437, 2006

2. Roberts E: Pediatric non-alcoholic fatty liver disease (NAFLD): a 'growing' problem? J Hepatol 46: 1133-1142, 2007.

3. Manco M, Bottazzo G, DeVito R, Marcellini M, Mingrone G and Nobili V: Nonalcoholic fatty liver disease in children. J Am Coll Nutr 27: 667-676, 2008.

4. Adams LA, Lymp JF, St. Sauver J, et al: The natural history of non-alcoholic fatty liver disease: a population-based cohort study. Gastroenterology 129: 113-121, 2005.

5. Nobili V, Marcellini M, Devito R, Ciampalini P, Piemonte F and Comparcola D: NAFLD in children: a prospective clinicalpathological study and effect of lifestyle advice. Hepatology 44: 458-465, 2006.

6. Adams LA, Sanderson S, Lindor KD and Angulo P: The histological course of non-alcoholic fatty liver disease: a longitudinally study of 103 patients with sequential liver biopsies. J Hepatol 42: 132-138, 2005.

7. Cortez-Pinto H, Caneiro de Moura M and Day CP: Nonalcoholic steatohepatitis: from cell biology to clinical practice. J Hepatol 44: 197-208, 2006.

8. Marra F, Gastaldelli A, Svegliati Baroni G, Tell G and Tiribelli C: Molecular basis and mechanisms of progression of nonalcoholic steatohepatitis. Trends Mol Med 14: 72-81, 2008.

9. Laurent A, Nicco C, Tran Van Nhieu J, et al: Pivotal role of superoxide anion and benefical effect of antioxidant molecules in murine steatohepatitis. Hepatology 39: 1277-1285, 2004.

10. Ronis MJ, Butura A, Korourian S, et al: Cytokine and chemokine expression associated with steatohepatitis and hepatocyte proliferation in rats fed ethanol via total enteral nutrition. Exp Biol Med 233: 344-355, 2008

11. Seki S, Kitada T, Yamada T, Sakaguchi H, Nakatani K and Wakasa K: In situ detection of lipid peroxidation and oxidative DNA damage in non-alcoholic fatty liver disease. J Hepatology 37: $56-62,2002$

12. Chalasani N, Deeg MA and Crabb DW: Systemic levels of lipid peroxidation and its metabolic and dietary correlates in patients with non-alcoholic steatohepatitis. Am J Gastroenterol 99: 1497-1502, 2004

13. Ikura Y, Ohsawa M, Suekane T, et al: Localization of oxidized phosphatidylcholine in non-alcoholic fatty liver disease; impact on disease progression. Hepatology 43: 506-514, 2006.

14. Sreekumar R, Rosado B, Rasmussen D and Charlton M: Hepatic gene expression in histologically progressive nonalcoholic steatohepatitis. Hepatology 38: 244-251, 2003. 
15. Nobili V, Manco M, Devito R, Ciampalini P, Piemonte F and Marcellini M: Effect of vitamin E on aminotransferase levels and insulin resistance in children with non-alcoholic fatty liver disease. Aliment Pharmacol Ther 24: 1553-1561, 2006.

16. Nobili V, Manco M, Devito R, et al: Lifestyle intervention and antioxidant therapy in children with nonalcoholic fatty liver disease: a randomized, controlled trial. Hepatology 48: 119-128, 2008.

17. Manco M and Nobili V: Intensive treatment and dietary fats in adolescents with nonalcoholic fatty liver disease. J Pediatr Gastroenterol Nutr 46: 224-230, 2008.

18. Manco M, Bedogni G, Marcellini M, et al: Waist circumference correlates with liver fibrosis in children with non-alcoholic steatohepatitis. Gut 57: 1283-1287, 2008.

19. Cole TJ, Bellizzi MC, Flegal KM and Dietz WH: Establishing a standard definition for child overweight and obesity worldwide: international survey. Br Med J 320: 1240-1243, 2000.

20. Genuth S, Alberti KG, Bennett P, et al: Expert Committee on the Diagnosis and Classification of Diabetes Mellitus. Followup report on the diagnosis of diabetes mellitus. Diabetes Care 26: 3160-3167, 2003.

21. Genuth S: Lowering the criterion for impaired fasting glucose is in order. Diabetes Care 26: 3331-3332, 2003.

22. Matsuda M and DeFronzo RA: Insulin sensitivity indices obtained from oral glucose tolerance testing: comparison with the euglycemic insulin clamp. Diabetes Care 22: 1462-1470, 1999.

23. Abdul-Ghani MA, Matsuda M, Balas B and DeFronzo RA: Muscle and liver insulin resistance indexes derived from the oral glucose tolerance test. Diabetes Care 30: 89-94, 2007.

24. Poynard T, Halfon P, Castera L, et al: FibroPaca Group: Variability of the area under the receiver operating characteristic curves in the diagnostic evaluation of liver fibrosis markers: impact of biopsy length and fragmentation. Aliment Pharmacol Ther 25: 733-739, 2007

25. Kleiner DE, Brunt EM, Van Natta M, et al: Nonalcoholic Steatohepatitis Clinical Research Network. Design and validation of a histological scoring system for nonalcoholic fatty liver disease. Hepatology 41: 1313-1321, 2005.

26. Novo E, Marra F, Zamara E, et al: Overexpression of Bcl-2 by activated human hepatic stellate cells: resistance to apoptosis as a mechanism of progressive hepatic fibrogenesis. Gut 55: 1174-1182, 2006.

27. Vidali M, Hietala J, Occhino G, et al: Immune responses against oxidative stress-derived antigens are associated with increased circulating tumor necrosis factor- $\alpha$ and accelerated liver damage in heavy drinkers. Free Rad Biol Med 45: 306-311, 2008.
28. Albano E, Mottaran E, Vidali M, et al: Immune response towards lipid peroxidation products as a predictor of progression of non-alcoholic fatty liver disease to advanced fibrosis. Gut 54: 987-993, 2005.

29. Hawkins CL, Morgan PE and Davies MJ: Quantification of protein modification by oxidants. Free Rad Biol Med 46: 965-988, 2009.

30. Molnar D, Decsi T and Koletzko B: Reduced antioxidant status in obese children with metabolic syndrome. Int J Obes Relat Metab Disord 28: 1197-1202, 2004.

31. Mohn A, Catino M, Capanna R, Giannini C, Marcovecchio M and Chiarelli F: Increased oxidative stress in prepuberal severely obese children: effect of dietary restriction-weight loss program. J Clin Endocrinol Metab 90: 2653-2658, 2005.

32. Nobili V, Pastrore A, Gaeta LM, et al: Glutathione metabolism and antioxidant enzymes in patients affected by non-alcoholic steatohepatitis. Clin Chim Acta 355: 105-111, 2005.

33. Mottaran E, Stewart SF, Rolla R, et al: Lipid peroxidation contributes to immune reactions associated with alcoholic liver disease. Free Rad Biol Med 32: 38-45, 2002.

34. Vidali M, Tripodi MF, Ivaldi A, et al: Interplay between oxidative stress and steatosis in the progression of Hepatitis C. J Hepatol 48: 399-406, 2008.

35. Albano E: Alcohol, oxidative stress and free radical damage. Proc Nutr Soc 65: 278-290, 2006.

36. Choi $\mathbf{J}$ and $\mathrm{Ou}$ JHJ: Mechanisms of liver injury III. Oxidative stress in the pathogenesis of hepatitis C virus. Am J Physiol 290: G847-G851, 2006.

37. Stewart SF, Vidali M, Day CP, Albano E and Jones DE: Oxidative stress as a trigger for cellular immune response in patients with alcoholic liver disease. Hepatology 39: 197-203, 2004.

38. Vidali M, Stewart SF and Albano E: Interplay between oxidative stress and immunity in the progression of alcoholmediated liver injury. Trends Mol Med 14: 63-71, 2008.

39. Chou MY, Hartvigsen K, Hansen LF, et al: Oxidation-specific epitopes are important targets of innate immunity. J Intern Med 263: 479-488, 2008.

40. Ma X, Hua J, Mohamood AR, Hamad AR, Ravi R and Li Z: A high-fat diet and regulatory $\mathrm{T}$ cells influence susceptibility to endotoxin-induced liver injury. Hepatology 46: 1519-1529, 2007.

41. George J: Mechanisms of disease: the evolving role of regulatory T cells in atherosclerosis. Nat Clin Pract Cardiovascular Med 5: 531-540, 2008. 